\title{
Towards a Platform for Smart Manufacturing Improvement Planning
}

\author{
SangSu Choi ${ }^{1}$, Thorsten Wuest ${ }^{2}$, and Boonserm (Serm) Kulvatunyou ${ }^{3}$ \\ ${ }^{1}$ IGI, LLC, Clarksburg MD 20871, USA \\ Sangsu.choiligiamerica.com \\ ${ }^{2}$ Industrial and Management Systems Engineering, West Virginia University, Morgantown, \\ WV 26506-6070, USA \\ thwuestemail.wvu.edu \\ ${ }^{3}$ Systems Integration Division, National Institute of Standards and Technology, Gaithersburg, \\ MD, USA 20899-1070, USA \\ boonserm. kulvatunyou@nist.gov
}

\begin{abstract}
The manufacturing industry is transitioning towards smart manufacturing systems (SMS). Small and medium size manufacturers (SMMs) are particularly behind in this transition, plagued by lack of knowledge and resources. Several smart manufacturing capability assessment and maturity models exist to guide the transition. However, support for choosing the right assessment is lacking. This paper proposes a web-based, open source platform for smart manufacturing assessment to support SMMs in this transition. The platform allows for free self-assessments of the current maturity levels and developments of continuous improvement plans that are customized to the manufacturers' unique characteristics. The platform also allows for sourcing of third-party technologies and services relevant to the improvements. More importantly, it will learn, rate, and recommend improvements and services based on past data. The platform is designed to be extensible and scalable to ultimately serve manufacturing enterprises of all industries.
\end{abstract}

Keywords: Smart Manufacturing, Smart Factory, Industrie 4.0, Cyber-Physical Production System, Smart Manufacturing Assessment, Smart Manufacturing Readiness

\section{Introduction}

The world is moving towards the next industrial revolution where manufacturing enterprises are seeking for performance improvements through a new level of interconnectivity within their own production environments and value chains. The core of the current industrial revolution is the development of smart manufacturing systems (SMS). SMS are built on the intersection of cutting-edge Information Technology (IT) and Operation Technology (OT) such as Internet of Things (IoT), big data, cloud 
computing, machine learning, additive manufacturing, $\mathrm{CNC}$ machine tools, and collaborative robots $[1,2]$.

A smart manufacturing system is ultimately realized by integrating several of these core technologies in a customized and value-adding way. SMS must be developed harmoniously based on a combination of IT/OT technologies that fit each individual company's unique manufacturing environment. A recent study by West Virginia University (WVU) [3] on Small and Medium-Size Manufacturers' (SMMs) smart manufacturing adoptions found that the vast majority of SMMs do not possess the required resources and are struggling in taking the critical first step of their own smart manufacturing journey. They need systematic assessment methodologies to better understand their needs and build a solid foundation for their own, value-adding SMS.

Several smart manufacturing and Industrie 4.0 manufacturing system assessment methods and capability maturity models are available. These methods and models evaluate a manufacturing enterprise from various perspectives with differing applicability to various sizes and maturity of the enterprise. There are also still limited wealth of information supporting the usages and derived values of these methods and models. Sorting through them will require expertise and resources SMMs typically lack. Using outside consultants can also be cost prohibitive and too risky for many SMMs.

This paper introduces a smart manufacturing improvement planning system that aims to systemize smart manufacturing consulting services, to be easily utilized by manufacturing companies. In the next section, related works are provided. Then, an overview of the platform is given, followed with discussion of the future work and finally conclusion.

\section{$2 \quad$ Related Works}

Over the past decade, frameworks or models for business and process maturity assessment have been developed within the manufacturing sector. Technology readiness level (TRL) [5] represents the maturity of a technology for commercial adoption. Similarly, manufacturing readiness level (MRL) [6] reflects the characteristics of manufacturing process technology. Supply chain readiness level (SCRL) [7] provides a way to assess the ability of the supply chain to operate and to achieve specific operational performance goals. It is associated with characteristics within fifteen categories that discretely provide an improvement roadmap for design and operation of a supply chain.

More recently as the new industrialization paradigms such as smart manufacturing and Industrie 4.0 have been developed, models for respective readiness and maturity assessments have also been proposed. Weber et al. [8] presented a model for data driven manufacturing in the context of Industrie 4.0 that consists of six maturity levels (0-Nonexistent IT Integration, 1-Data and System Integration, 2-Integration of Cross-Life-Cycle 3-Data and Service-Orientation, 4-Digital Twin, 5-Self-Optimizing Factory). Lee et al. [9] developed an assessment framework in the context of smart factory that consists of Leader-ship, Process, System and Automation, and Perfor- 
mance Criteria. In addition, they introduced the effects based on 20 case studies of South Korean SMEs.

Carolis et al. [10] introduced the Digital REadiness Assessment MaturitY (DREAMY) model that focuses more on the digitalization of an enterprise. It aims to assess a manufacturing enterprise readiness level for the digital transformation and to develop a roadmap for prioritizing investments. Smart manufacturing system readiness level (SMSRL) [4] was developed at the National Institute of Standards and Technology (NIST) as an index that measures a manufacturing company's readiness for improving manufacturing system performance using data intensive smart manufacturing technologies. It measures the readiness in four aspects including ICT, performance management, organization, and information connectivity. Li et al. [11] introduced an assessment model using MESA MOM Capability Maturity Model (CMM) [12] and suggested a way to reduce the time and resources consumption for applying the CMM to manufacturing enterprises by replacing the lengthy yes/no questionnaires with multiple-choice and providing various kinds of improvement strategies.

Although various smart manufacturing assessment models are being developed, their applicability issues remain, particularly regarding SMMs special requirements. SMMs typically need trainings to conduct assessments internally. Alternatively, consulting services may be used. Both options add more loads to the SMMs scarce resources. For these reasons, SMEs need a clear value proposition and a mechanism to evaluate the trustworthiness of consulting services.

To solve these problems, it is necessary to develop an assessment platform that enables manufacturers to either conduct a self-assessment with a very intuitive, step-bystep user interface, or provide access to assessment models and community-evaluated consulting services. The platform should allow users to develop subsequent improvement plans and share success values.

\section{Smart Manufacturing Assessment System}

\subsection{SMAS Architecture}

This assessment system is being developed based on the MEAN stack (MongoDB, Express.js, Angular 4, and Node.js). It will be an open source, web-based, free selfassessment system for manufacturing companies. It will provide a platform to host various SMS assessment methodologies [e.g., 4, 8, and 9], reflecting the diversity of manufacturing companies and their individual, often domain specific requirements. Manufacturing enterprises can intuitively compare their current smart manufacturing readiness to reference models and peers, adopt improvement strategies, get technologies and services recommendations that have been community-vetted, and generate improvement roadmap systemically on the platform.

Fig. 1 shows the system architecture. Professionals can add assessment methods into SMAS by a convenient procedure. A bundle that consists of a detailed description, questionnaires, computation logics, and charts are defined using a standard XML schema shown in Fig. 2 (a). The mounting of an assessment model is completed up- 
loading XML files using the neutral language interface of SMAS. Manufacturing users select one of the assessment models to conduct their assessment as shown in Fig. 2 (b). Intuitive charts indicating the current status are provided as shown in Fig. 2 (c). Improvement plans are automatically presented based on the assessment results. Administrators can analyze accumulated data and generate industry, regional, or national level benchmarking reports without revealing individual manufacturer's identity.

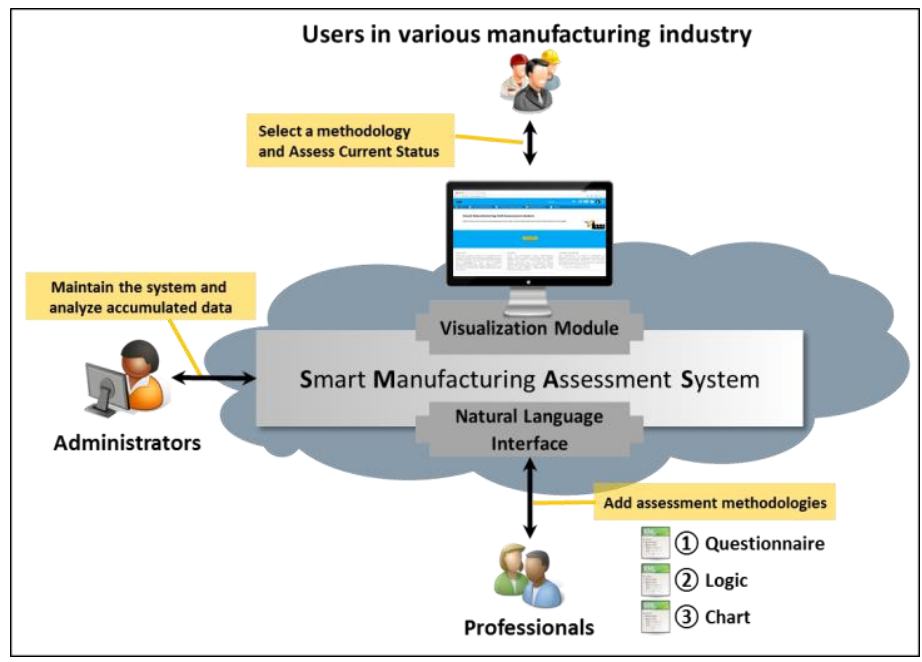

Fig. 1. Architecture of SMAS

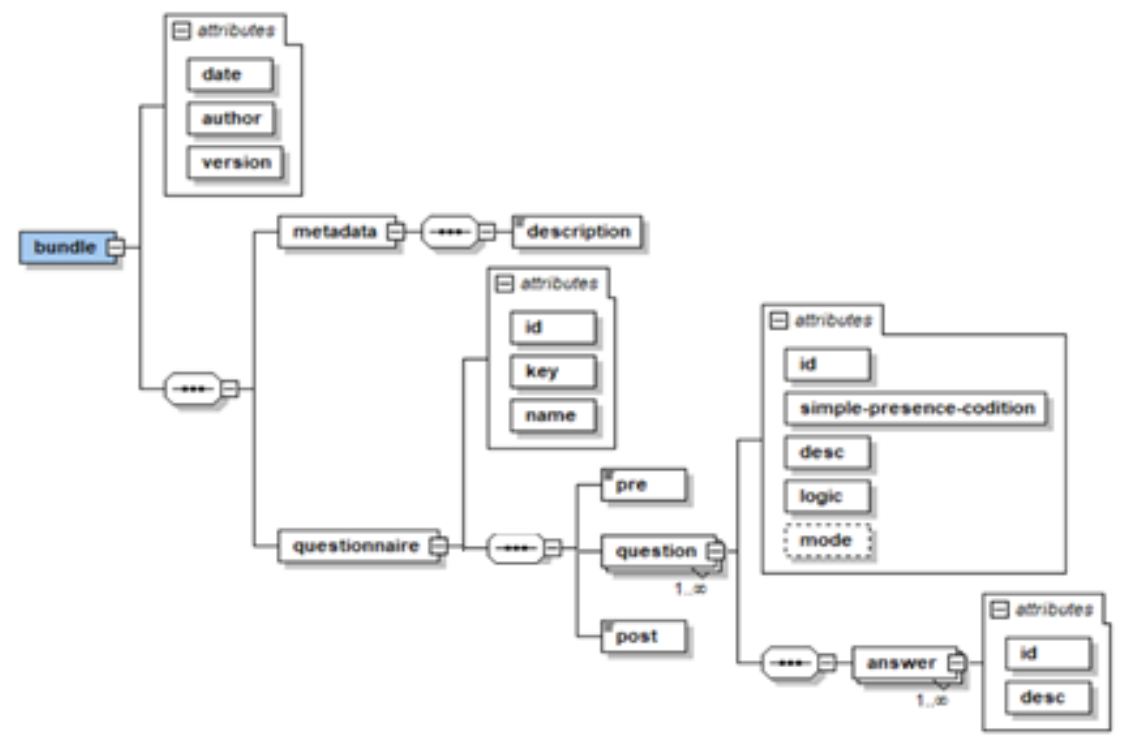


(a) XML Schema

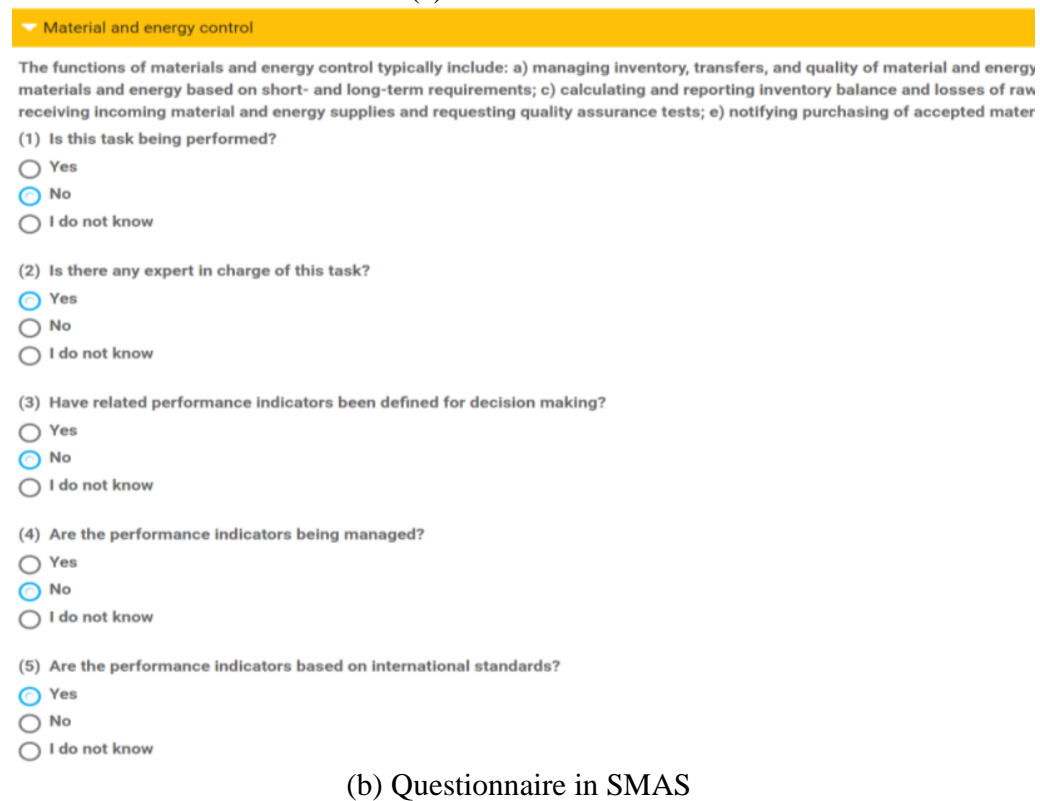

(b) Questionnaire in SMAS

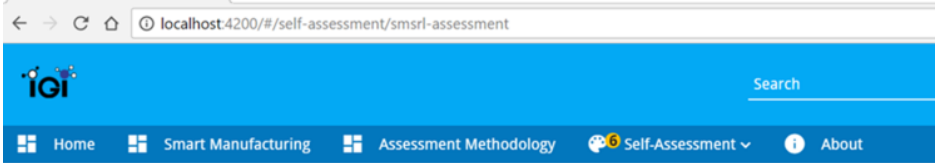

1. Smart Manufacturing Maturity Index
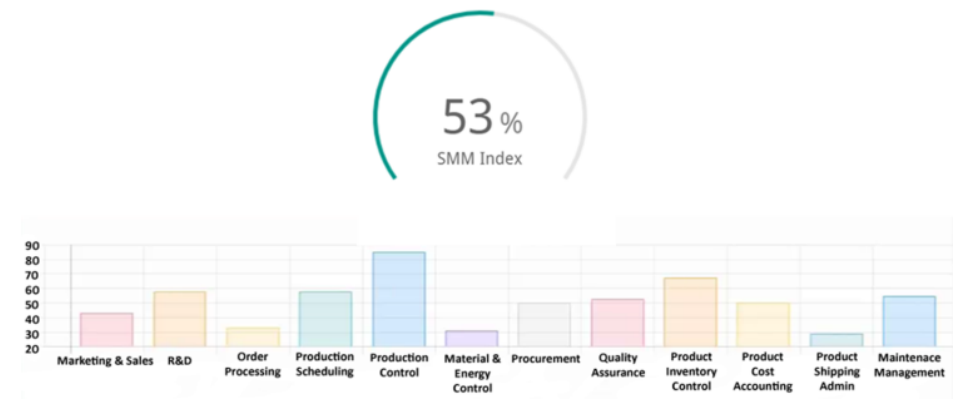

(c) Charts in SMAS

Fig. 2. XML Schema for assessment models and SMAS user interfaces

\subsection{Assessment Methodologies}

Two assessment methods are currently equipped in SMAS prototype. The first model is SMSRL [4] that seeks to provide an evaluation of how manufacturers manage/improve their performances and the available infrastructure to support related activities. SMSRL method is based on a formal reference model for factory improvement activities called FDI [13] and evaluates manufacturers in four aspects including 
organizational maturity, performance management maturity, IT maturity, and information connectivity maturity as shown in Fig. 3. Consolidating these four categories into a single measure creates a maturity index reflecting a manufacturer's readiness to deploy smart manufacturing solutions or to participate in a network of smart manufacturers.

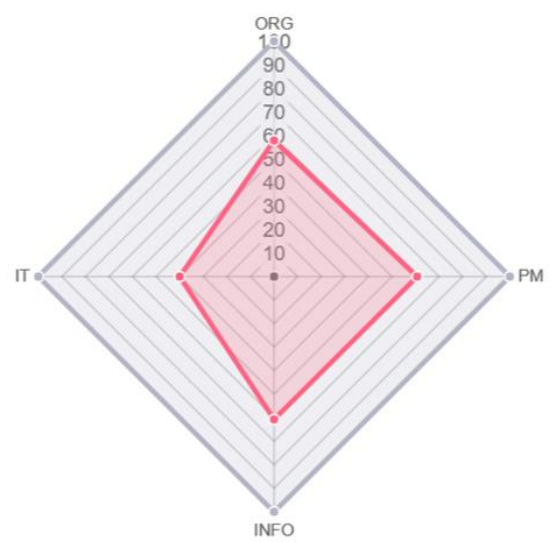

Fig. 3. Four aspects of SMSRL

The second unique methodology is developed by WVU's Smart Manufacturing Lab and focuses specifically on the needs of manufacturing SMEs. The WVU assessment and maturity model is unique in several aspects: i) it reflects the lower baseline several manufacturing SMEs have when it comes to Smart Manufacturing (adding a 'level $\left.0^{\prime}\right)$, ii) it merges a readiness index with a maturity model, and iii) integrate building blocks and toolkits that can be combined in a modular fashion based on the individual maturity and assessment outcome. The model will be set around five organizational dimensions and five maturity levels. The model is informed by a recently conducted in-depth study of the current state of the art of Industrie 4.0 / Smart Manufacturing maturity/ assessment/ readiness models and the learning from WVU's recent SME study [3].

\subsection{Discussion}

The choices of assessment methods included in SMAS prototype validated its scalability to address different needs varied by sizes and maturities of manufacturers. The SMSRL method is applicable to a more mature typically of medium to large sizes manufacturer, while the WVU method is applicable to a less mature typically of a small size manufacturer. Going forward, SMAS will provide user-friendly interfaces (UI) for professional or admin users to conveniently add various assessment methods. SMAS will be developed as a scalable open source platform with a high degree of openness and collaboration as shown in Fig. 4 [14]. Manufacturing users will intuitively compare different SMS technologies and services on the platform. Suitable solutions are matched with individual manufacturers requirements by a machine learning algorithm. The machine learning will use data from a peer review system and 
success stories associated with the past technologies and services adoptions. Thereby the effectiveness of suggestions will improve with scale and over time. SMAS will allow manufacturers to assess their smart manufacturing level for free by themselves. Guidelines including tools, advice and counsel on how to invest wisely will be provided separately by industry.

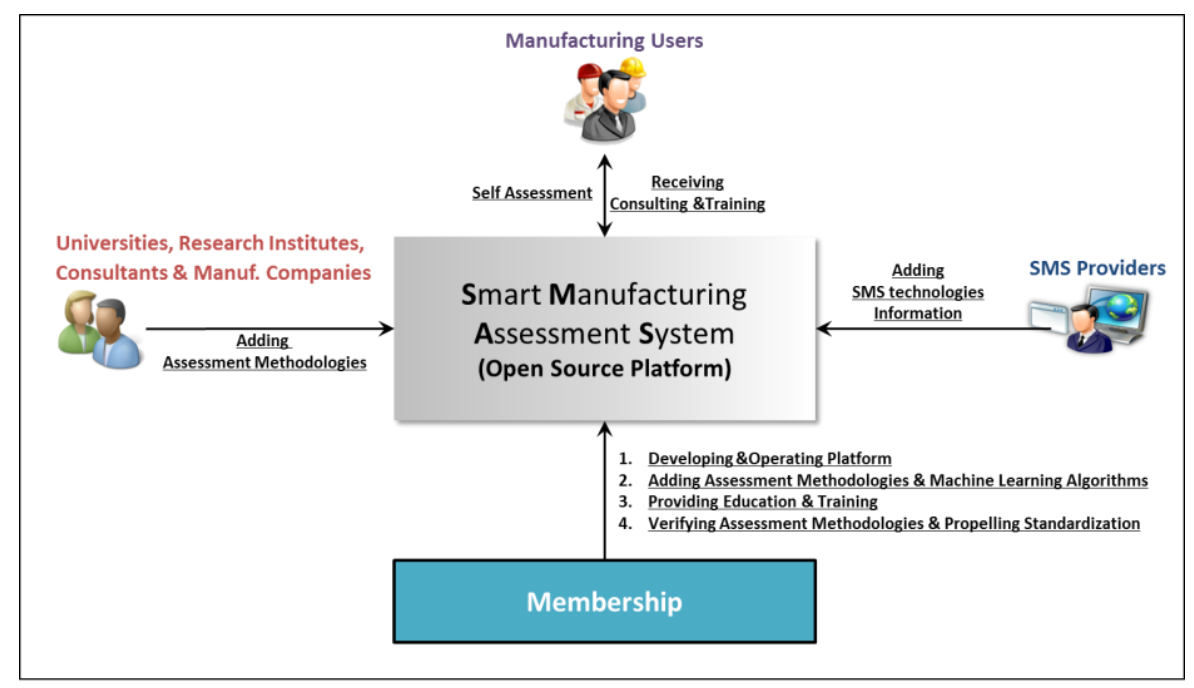

Fig. 4. Concept of Platform

SMAS services will sustain by charging advertising and/or transaction fees from trainings, consulting services, technologies offered on the platform. They will be propelled by developments and standardizations of assessment methodologies and increasing utilizations.

\section{Conclusions}

A smart manufacturing assessment system called SMAS for manufacturing enterprises, especially SMMs, is being developed. SMAS will be released in the near future as an open source platform. Through this platform, technologies, knowledge and information related to SMS will be widely shared. It will also provide appropriate assessment methodologies and best practices for various industries and national users. Accumulated big data from manufacturing users will be analyzed, and it can be used for developing appropriate government support policy and SMS technology roadmap. This platform will help manufacturing companies improve SMS understanding and build their own smart factories. Creating a coherent suite of assessment methods or showing their differences and relationships remain a challenge. In our future work, we plan to investigate the possibility of using the three smart manufacturing dimensions, namely smart factory, value chain, and digital thread proposed by [15] as a guidance. 


\section{Disclaimer}

Any mention of commercial products is for information only; it does not imply recommendation or endorsement by NIST.

\section{References}

1. Thoben, K. D., Wiesner, S., Wuest, T.: Industrie 4.0 and Smart Manufacturing-A Review of Research Issues and Application Examples, International Journal of Automation Technology 11(1) (2017).

2. Kang, H. S., Lee, J. Y., Choi, S., Kim, H., Park, J. H., Son, J. Y., Noh, S.D.: Smart manufacturing: Past research, present findings, and future directions. International Journal of Precision Engineering and Manufacturing-Green Technology 3(1), 111-128 (2016).

3. Wuest, T., Schmid, P., Lego, B. \& Bowen, E.: Overview of Smart Manufacturing in West Virginia. Bureau of Business \& Economic Research West Virginia University.

4. Jung, K., Kulvatunyou, B., Choi, S., Brundage, M. P.: An Overview of a Smart Manufacturing System Readiness Assessment. In IFIP International Conference on Advances in Production Management Systems, pp. 705-712. Springer, Cham (2016).

5. Dubos, G. F., Saleh, J. H., and Braun, R.: Technology readiness level, schedule risk, and slippage in spacecraft design. Journal of Spacecraft and Rockets 45(4), 836-842 (2008).

6. Ward, M. J., Halliday, S. T., and Foden, J.: A readiness level approach to manufacturing technology development in the aerospace sector: an industrial approach. Proceedings of the Institution of Mechanical Engineers, Part B: Journal of Engineering Manufacture 226(3), 547-552, (2012).

7. Tucker, B., and Paxton, J.: SCRL-model for human space flight operations enterprise supply chain. In Aerospace Conference 2010, pp.1-9. IEEE (2010).

8. Weber, C., Königsberger, J., Kassner, L., Mitschang, B.: M2DDM-A Maturity Model for Data-Driven Manufacturing. Procedia CIRP, Vol.63, pp.173-178 (2017).

9. Lee, J., Jun, S., Chang, T. W., Park, J.: A Smartness Assessment Framework for Smart Factories Using Analytic Network Process. Sustainability, 9(5), 794 (2017).

10. De Carolis, A., Macchi, M., Negri, E., Terzi, S.: A maturity model for assessing the digital readiness of manufacturing companies. In IFIP International Conference on Advances in Production Management Systems, pp.13-20, Springer, Cham (2017).

11. Li, Q., Brundage, M., Kulvatunyou, B., Brandl, D., Noh, SD.: Improvement Strategies for Manufacturers Using the MESA MOM Capability Maturity Model. In IFIP International Conference on Advances in Production Management Systems, pp. 21-29, Springer, Cham (2017).

12. D. Brandl, "MESA MOM Capability Maturity Model Version 1.0," White Paper \#53, MESA International white paper, 2016.

13. Jung, K., Choi, S., Kulvatunyou, B., Cho, H., Morris, K. C.: A reference activity model for smart factory design and improvement. Production Planning \& Control 28(2), pp.108-122 (2017).

14. Menon, K., Kaerkkaenen, H., Wuest, T.: Role of openness in industrial internet platform providers' strategy. PLM 2017, Seville, Spain (2017).

15. Leiva, C. (February 2018). Three perspectives converge on smart manufacturing. Internet article available at http://advancedmanufacturing.org/17797-2/. 